\title{
Interview with Yakov Sinai
}

Martin Raussen and Christian Skau

Yakov Sinai is the recipient of the 2014 Abel Prize of the Norwegian Academy of Science and Letters. This interview was conducted by Martin Raussen and Christian Skau in Oslo on May 19, 2014, in conjunction with the Abel Prize celebration. This article originally appeared in the September 2014 issue of the Newsletter of the European Mathematical Society and is reprinted here with permission of the EMS.

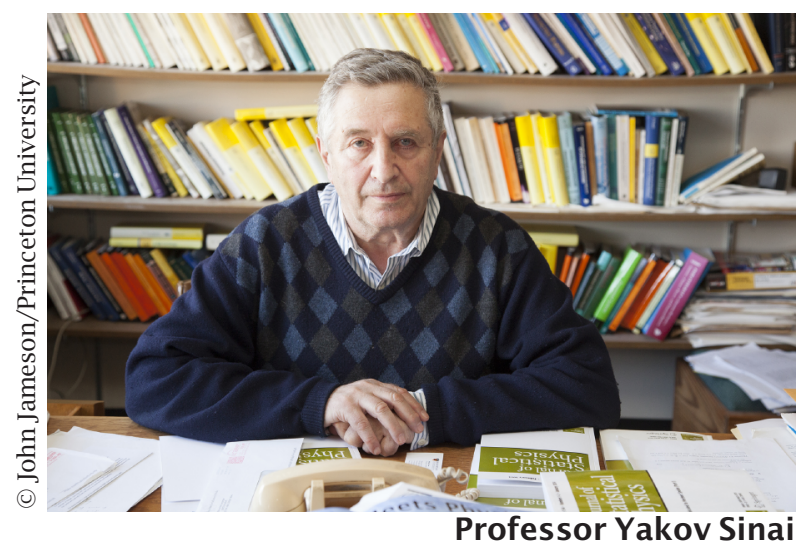

The Prize

Raussen and Skau: Professor Sinai, first of all we would like to express our congratulations. You have been selected as the twelfth winner of the Abel Prize and you will receive the prize tomorrow. We are curious; did you have any expectations beforehand? How did you receive the information?

Professor Sinai: In early March this year I got to know that the Abel Committee was interested in taking my photograph. A friend of mine told me this, and I thought this must mean something because this had never happened before. And then there was a telephone call from the Norwegian Academy of Science and Letters informing me about the prize.

Raussen and Skau: And this was on the same day that the prize was announced here in Oslo?

Martin Raussen is associate professor of mathematics at Aalborg University, Denmark. His email address is raussen@math . aau.dk.

Christian Skau is professor of mathematics at the Norwegian University of Science and Technology, Trondheim, Norway. His email address is csk@math.ntnu. no.

DOI: http://dx.doi.org/10.1090/noti1210
Professor Sinai: Yes. That happened on March 26.

\section{Youth}

Raussen and Skau: You were born in Moscow in 1935 into a family of scientists. Your parents were both biologists, and your grandfather was well known in mathematics. We suppose this had important consequences for the development of your interests?

Professor Sinai: Definitely, yes. How could I say no to this question? Everything was about mathematics and mathematical events. But, at that time, I preferred to play volleyball.

The influence of mathematics was not as direct as you may think. I participated in many olympiads in mathematics during my school years but never had any success and never won any awards. I say this to young people who have never won in olympiads; there may be compensation in the future.

At this time, my grandfather was of a great age and he did not have the energy to push me into mathematics. And I also have a half-brother, G. I. Barrenblatt, who worked at Moscow State University and who was convinced that I should pursue a career in mathematics.

Raussen and Skau: Do you remember when you found out that you had an exceptional talent for mathematics?

Professor Sinai: If at all, it happened very late. I was a graduate student when I brought my paper on entropy to my advisor, A. N. Kolmogorov and he said, "At last you can compete with my other students." But I am not sure that he was right and that I have an exceptional talent for mathematics.

Raussen and Skau: You must have entered school at about the same time that Nazi Germany invaded Russia. How did the war influence your first years at school? 
Professor Sinai: I entered school in 1943 after my family returned from the evacuation of Moscow. At that time boys and girls studied separately; at the end of each year, we had about ten exams. Before the evacuation, life was different. It was forbidden to leave windows open in the apartments in Moscow because it had to be dark. In 1943 windows were allowed to be open again. In Moscow there were no clear signs of war, but life was hard because of the time of Stalin. People had to behave in a special way.

Raussen and Skau: And that also influenced life at school?

Professor Sinai: It was everywhere; you could be expelled from school or even sent to prison for being controversial.

Raussen and Skau: Were there teachers with a lot of influence on you, in particular in mathematics?

Professor Sinai: We had a very good teacher in mathematics at our high school. His name was Vasily Alekseevich Efremov and he was a great old-style schoolteacher. He always brought us his problems in accurate handwriting on a piece of paper which he distributed among the students. Because of the well-organized and inspiring work, mathematics was very popular among us. We discussed and tried to solve his problems. At this time I was not among the best in the class. There were definitely other students who were much better than I.

Raussen and Skau: What was your age at this point?

Professor Sinai: This was still in high school just before I entered university. Thus, I was probably sixteen or seventeen years old.

\section{Student at MSU-Mech-Mat}

Raussen and Skau: You entered the Faculty of Mechanics and Mathematics at Moscow State University in 1952 still a teenager. How was it to study at this famous institution as such a young student?

Professor Sinai: We had a number of very good professors there. For example, the lecture course in analysis was given by M. A. Lavrentiev, who was a very famous scientist at the time. He was also involved in administration but was a great teacher and his lectures were very interesting. We also had a very good lecturer in classical mechanics, Chetaev. I was his student in the second year. Moreover, we had lectures in geometry given by Bachvalov, who was famous in Russia but not so much known in the West. There is a story about him: when we entered the university on 1 September, he came into the room and said, "Let's continue." And that was the beginning of his lecture course.

In algebra we had lectures by Dynkin, who was an excellent teacher for those who had started to study. These were lectures at a very high level. Dynkin used to hand out interesting problems for the enthusiastic students. Among such students in my year I could particularly mention I. Girsanov, who became a famous probabilist, and L. Seregin.

Raussen and Skau: Was it Dynkin who inspired your first paper in mathematics?

Professor Sinai: Yes. I was a student of Dynkin during the second and third years and I wrote the first paper under his supervision. I solved a problem that he formulated for me; this became my first published paper when I was a student in the third year. I loved the work I did and still do.

Dynkin wanted me to work on problems on Markov processes in the style of Feller. The papers by Feller became very popular in Moscow at that time, and Dynkin suggested that I should continue along this line. However, I was not very excited and interested in it.

Raussen and Skau: To what extent were mathematics and mechanics integrated in the curriculum?

Professor Sinai: These were independent parts of the curriculum. Everybody could attend lectures within each branch. I was attending lectures in mathematics and mechanics but also, to a minor degree, some lectures in physics. But on the whole it was mainly in the mathematics department.

Raussen and Skau: We imagine that besides Dynkin, Kolmogorov and Gelfand must have been very important figures for you?

Professor Sinai: Kolmogorov had many students, and I became one of them. His students had complete freedom to work on any problem. Kolmogorov loved to discuss their results. There were several cases when Kolmogorov wrote their papers in order to teach them how to write mathematical texts.

Kolmogorov organized a seminar, which was initially a seminar on random processes and later became a seminar on dynamical systems and ergodic theory. I began to attend, together with other mathematicians like Arnold, Alekseev, Tikhomirov, and others. Later I became a student of Kolmogorov. At that time he was also interested in problems of entropy in different compact and functional spaces. Questions of this type were very much discussed at that time.

Raussen and Skau: But Gelfand tried to recruit you as a graduate student as well?

Professor Sinai: Yes. Gelfand organized his famous seminar, which was attended by many mathematicians of different generations. I took part in it for many years. It happened, if I remember it correctly, in 1955 when Gelfand was writing a famous volume in his series of books on distributions. Gelfand was interested in probability theory and he wanted me to become his student. We had some discussions about it, and I told him that I wanted very much to work on problems related to probability theory but I had already written a paper under the supervision of Dynkin. He asked me, "Do you want to have probability theory as an 
appetizer or as a dessert?" I answered, "I want it as a main course." That was the end of the story....

This did not mean that our contact came to an end; we met many times, especially when he worked on problems in representation theory, which were connected with problems in ergodic theory, like the theory of horocycles and others. We discussed this many times. I attended Gelfand's seminars for many years, because Gelfand had the remarkable ability to explain difficult mathematical topics in a clear and simple way.

\section{Dynamical Systems. Entropy and Chaos}

Raussen and Skau: Could you explain what a dynamical system is?

Professor Sinai: We understand dynamical systems as objects that describe all types of evolution. The most interesting case is nonlinear dynamical systems, when the formulas for the dynamics of the evolution are nonlinear. There can be many different phenomena which require deep analysis.

Raussen and Skau: And among these dynamical systems, what is an ergodic system?

Professor Sinai: I have a very good example for an ergodic system which I always explain to my students. Suppose you want to buy a pair of shoes and you live in a house that has a shoe store. There are two different strategies: one is that you go to the store in your house every day to check out the shoes and eventually you find the best pair; another is to take your car and to spend a whole day searching for footwear all over town to find a place where they have the best shoes and you buy them immediately. The system is ergodic if the result of these two strategies is the same. The entropy characterizes the growth of the number of possibilities in dynamics. I heard the first explanation of this role of entropy from I. M. Gelfand.

Raussen and Skau: Ergodic theory originally came from physics, in particular from the study of Hamiltonian equations. Can you explain in general terms what chaos is and how one can measure it?

Professor Sinai: This is the subject of my lecture, which I will give the day after tomorrow, but I can summarize it briefly here. The main question concerns the difference between chaos theory and probability theory. In probability theory one deals with statistical experiments: say you toss a coin 100 times. One can have many different series resulting from this experiment and study the result.

If you consider the problem of chaos and, for example, want to measure the temperature at the same point you make the measurement during the year, you now have only one realization of the temperature. You cannot have a hundred realizations of the temperature at a given place and at a given time. So the theory of chaos studies the series when the results of measurements have a limit as time tends to infinity and how to describe this limit. The existence of the limit actually follows from some hypothesis about the equations of motion. This gives the existence of the distribution, which determines the value of all kinds of averages (or, it is better to say, the existence of the averages and also finding their values).

Then, the question is: What are the equations of motion which determine the distribution and these averages? The basic statement in chaos theory is that the dynamics must be unstable. Instability means that small perturbations of the initial conditions lead to large perturbations in the dynamics after some time.

Then there is a mathematical theory that says that if the system is unstable, the time averages exist and there is a possibility of calculating them. This is the general description of what is done in chaos. A more precise description requires more mathematics.

Raussen and Skau: How do you measure chaos? Does entropy come into the picture here?

Professor Sinai: If we understand chaos as mentioned already, i.e., as the existence of time averages and also properties related to mixing, then there is a natural description of chaos in terms of some special distributions. Entropy is used in the theory of unstable systems and it characterizes how many types of dynamics a given system can have. It is certainly a very useful notion, because the positivity of the entropy determines other properties of the systems that can be studied.

Physicists always expressed their hope that entropy would allow them to understand turbulence (see, for example, the paper by B. Chirikov and the books by A. Zaslavski, R. Sagdeev, and others). It is hard to say that this hypothesis is true. On the other hand, there are many situations in physics where systems have small entropy.

\section{Definition of Entropy for Dynamical Systems}

Raussen and Skau: Kolmogorov had come up with the definition of entropy for Bernoulli shifts, but then he changed it to a definition that was not invariant. Then you came with the correct definition. What is now called the Kolmogorov-Sinai theorem gives an efficient way to compute the entropy.

Professor Sinai: Kolmogorov started his seminar with von Neumann's theory of dynamical systems with pure point spectrum, which he explained in a purely probabilistic way. Later I found this approach in the book by Blanc-Lapierre and Fortet. Everything in Kolmogorov's seminars was very exciting. At that time we believed that the main problem in ergodic theory was to extend the theory of von Neumann to systems with continuous spectrum that can be constructed in terms of the second homology group of the spectrum with coefficients in the ring of bounded operators. It did not work, but the idea remained. 
At that time, Kolmogorov spent his time primarily on problems in information theory and the concept of dimension of linear spaces. I do not know how it happened, but one day Kolmogorov came to his lecture and presented his definition of entropy. Using modern terminology, one can say that he gave the definition of entropy for Bernoulli shifts and thus proposed a new invariant for this class of dynamical systems. It was certainly a great result. Kolmogorov wrote his text. He submitted it for publication and left for Paris where he spent the whole semester. As is known, the text that was submitted for publication was different from what he explained in class. In his paper he introduced a new class of systems which he called quasi-regular. Later they were called K-systems (K for Kolmogorov). For this class of systems he introduced the notion of entropy. While Kolmogorov was away, I was thinking about a definition of entropy that could work for all dynamical systems. Later it appeared in my paper on entropy.

At that time, there was a clear feeling that for dynamical systems appearing in probability theory, the entropy is usually positive, while for dynamical systems generated by ODEs it should be zero. Thus, there seemed to be a possibility to distinguish dynamical systems in probability theory from dynamical systems in analysis.

Raussen and Skau: How about your connection with Rokhlin?

Professor Sinai: The story about my connection with Rokhlin, who later became a close friend of mine, started when Kolmogorov's paper on entropy appeared in 1958. At that time, Rokhlin lived in a small provincial town, Kolomna, not far from Moscow. He had a very good graduate student Leonid Abramov. There are several general theorems that were proven by Abramov, like the entropy of special flows and other things like Abramov's formula, etc. When Rokhlin heard about the paper by Kolmogorov, he sent Abramov to Moscow to find out what had really been done, what was the situation, and if possible to bring the text.

When Abramov came to Moscow, he found me; we talked a lot, and I taught him everything I knew. Abramov then invited me to Kolomna to talk to Rokhlin, and I accepted the invitation. I remember my first visit to Kolomna very well. Rokhlin had an apartment there, which was very orderly; everything was very accurate, and he was dressed very well. We began to talk, and he made a very strong impression on me.

Rokhlin had great experience in ergodic theory because he had already published several papers in this field. His doctoral thesis was also about this subject. Rokhlin formulated a number of interesting problems in ergodic theory. Some of them were connected with Rokhlin's theory of measurable partitions. This theory became very useful in ergodic theory, because through it one can understand conditional probabilities in probability theory much better.

One of the problems that I began to work on under the influence of Rokhlin was the calculation of entropy for group automorphisms of the twodimensional torus. At that time it was not known that Kolmogorov's definition had to be modified; the analysis was rather difficult, and I could not achieve anything. Following the ideology of that time, I tried to prove that the entropy was zero, but all my attempts failed. Then I visited Kolmogorov and showed him my drawings. He said that it was clear in this case that the entropy must be positive. After that I proved the result.

At that time there was no question about publication of my paper, because Kolmogorov's paper on entropy had been published and it was not clear why another definition of entropy was needed. However, after some time, Rokhlin pointed out his result about the deficiencies in the definition by Kolmogorov. It became clear that I had to publish my paper with the definition and the calculation of the entropy for the automorphism which I had already done at that time.

This was the beginning of my contact with Rokhlin. After that he organized a seminar on ergodic theory in Moscow, which was attended by Arnold, Anosov, Alexeev, and others. In parallel, he had a seminar in topology where Novikov was the central figure.

Later Rokhlin moved to Leningrad (Saint Petersburg), and I used to go there to give talks at his seminar on later results.

\section{Billiard Systems}

Raussen and Skau: You then came up with an extremely interesting example of an ergodic system, the so-called billiards. Can you explain what these are?

Professor Sinai: A billiard, as people know, is the motion of a ball on the billiard table. An interesting mathematical theory arises if you allow the table to have a more or less arbitrary form. A natural question, which was actually raised by the Russian physicist Krylov long before the theory of entropy appeared, was: Which billiard systems have the same instability as the dynamics of particles moving in a space of negative curvature? Particles moving in a space with negative curvature yield the best example of unstable systems. The theory of billiards says that if the boundary of the table is concave, then the system is unstable in the sense we previously described. If we consider two initial conditions with different values of the velocities, then the corresponding trajectories diverge exponentially. If you consider a typical unstable billiard, namely the usual square billiard with a circle removed, then the difference between this billiard and the usual billiard is that for the 
unstable billiard the particles come to the holes much faster than for the usual billiard.

Raussen and Skau: This may become a little technical now. You proved a very important result about systems with positive entropy. Given a system with positive entropy, you can find a Bernoulli shift, which is a so-called factor, with the same entropy. This implies that if you have two Bernoulli shifts with the same entropy, they are what is called weakly isomorphic. Ornstein proved later that entropy is a complete invariant for Bernoulli shifts. It follows then from the work of Ornstein that the billiard example is the most chaotic system and is actually a Bernoulli flow, right?

Professor Sinai: From Ornstein's theorem it follows that if we have two ergodic billiard systems with the same value of entropy, then they are isomorphic. This is a remarkable and great result.

Raussen and Skau: So coin tossing is, in a sense, similar to the deterministic billiard system-an amazing fact.

Professor Sinai: My result says that if you have a system with positive entropy, there could be subsystems that move like Bernoulli shifts.

Raussen and Skau: What about billiard systems in higher dimensions? Is anything known there?

Professor Sinai: A lot of things are known. We have, for example, the result from the Hungarian mathematician Nåndor Simånyi, who is in Alabama now. He studied multidimensional dynamical systems that eventually become unstable and have positive entropy and are ergodic.

Raussen and Skau: You introduced Markov partitions in your study of Anosov diffeomorphisms. This led to what later became known as the SinaiRuelle-Bowen measure, also referred to as the SRBmeasure. Would you please explain?

Professor Sinai: First of all, there was my paper where I constructed this measure for the case of the so-called Anosov systems, or just hyperbolic systems. Then there was a paper by Bowen and Ruelle where they extended this construction to systems considered by Smale, that is, Axiom A systems with hyperbolic behavior.

These measures are important if you study irreversible processes in these systems. Suppose you start with some nonequilibrium distribution and consider the evolution and you ask how a nonequilibrium distribution converges to the equilibrium one. The result of the theory says that the evolution is in a sense very nonuniform, along some directions the expansion is very small and all the time averages behave very well and converge to a limit. But along other directions this convergence is very erratic, and hence it can only be studied using probability theory. So the measures, which are called SRB-measures, are the ones which are smooth along some unstable directions and are very irregular along other directions. This is a class of measures that appears in the theory of evolution of distributions in the case of chaotic systems.

Raussen and Skau: Are the SRB-measures related to Gibbs measures?

Professor Sinai: Yes. These measures are examples of Gibbs measures, but the Gibbs measures are much more general objects.

\section{Mathematics and Physics}

Raussen and Skau: Let's go back to more general questions, starting with the interplay between mathematics and physics. May we begin with the physicist Eugene Wigner, who in 1960 published the paper "The unreasonable effectiveness of mathematics in the natural sciences," in which he gave many examples showing how mathematical formalism advanced physical theory to an extent that was truly amazing? Do you have a similar experience?

Professor Sinai: My impression is that this effectiveness of mathematics is no longer a surprise for people. There are so many cases, for instance, the fact that string theory is practically a mathematical theory for physics. Some time ago Joel Lebowitz organized a discussion about this phrase of Wigner, in particular, how it can be that mathematics is so effective. The conclusion was that this is just a well-established fact.

In my generation, there was a group of young mathematicians who decided to study physics seriously. However, there were different points of view of how to do mathematical physics. F. A. Berezin always stressed that mathematicians should prove only results that are interesting for physicists. R. L. Dobrushin and I always tried to find in physical results some possibilities for mathematical research.

Raussen and Skau: On the other hand, there seems to be influence going in the opposite direction. Physicists have had a noteworthy impact on questions in quantum geometry and sometimes even in number theory. They have come up with formalisms that were not really developed in mathematics but nevertheless led to correct predictions which could be verified only after lengthy mathematical development.

Professor Sinai: So mathematics is effective, but you can say that it is not effective enough.

Raussen and Skau: You published in 2006 an article with the title "Mathematicians and Physicists = Cats and Dogs?" What is the main message of that paper?

Professor Sinai: I wanted to show examples where mathematicians and physicists look at the same problems differently. One example for this is the following story: my student Pirogov and I worked on problems in the theory of phase transitions in statistical physics. We proved several theorems, and I went to meet the famous Russian physicist Ilya Lifshitz to show him our theory; Lifshitz replaced Lev Landau when Landau had his 
car accident, severely incapacitating him. When I presented the theory he stopped me and said, "It's very simple what you are talking about." He started to write formulas which eventually gave our results. I left him very much embarrassed and I started to think why this had happened. I realized that the final result of our theory was an obvious statement for him. He certainly did not know how to prove it, but he did not need the proof. He just used it as an obvious fact.

Raussen and Skau: There is a famous quotation of the great Gauss: "Now I have the result. The only thing remaining is the proof." So intuition does play an important role in mathematics...

Professor Sinai: I can also tell the following story, again connected to Gelfand. I explained to him a theorem, which we obtained together with Robert Minlos. And Gelfand said, "This is obvious. All physicists know this." So we asked him if it was so obvious, should we write a text of 200 pages with complete proofs? He looked at us and said, "Certainly, yes!"

\section{A Jewish Mathematician in the Soviet Union}

Raussen and Skau: May we continue with a political question? You mentioned that being at school in the time of Stalin was not easy; life was still difficult for you when you entered university and started your career. You came from a Jewish family; in the Soviet Union, at least sometimes, a latent antiSemitism prevailed....

Professor Sinai: I can mention two cases in my career when I encountered anti-Semitism. The first one was the entrance examination, which I failed. The influence of my grandfather, who was head of the Chair of Differential Geometry, and the help from the President of Moscow University, I.G. Petrovsky, were needed in order to give me the possibility of being admitted to the University. This was a clear sign that things were not simple.

The other case arose with my entrance examination to graduate school. This exam was about the history of the Communist Party; I was very bad in this topic and failed the exam (I don't want to discuss the details). But P. S. Alexandrov, who was head of the mathematical department at Moscow State University, together with Kolmogorov, visited the head of the Chair of the History of the Party and asked her to allow me to have another attempt. She gave permission, and I got a B on the second attempt, which was enough to enter the graduate school. The result was not clear a priori, and it could have gone either way.

Raussen and Skau: In spite of these obstructions, it is quite obvious that many famous Russian mathematicians were and are of Jewish origin. This is quite amazing. Can you offer any explanation?

Professor Sinai: First something trivial: Jews had more traditions in learning than other nations. They study the Bible, the Talmud, and other

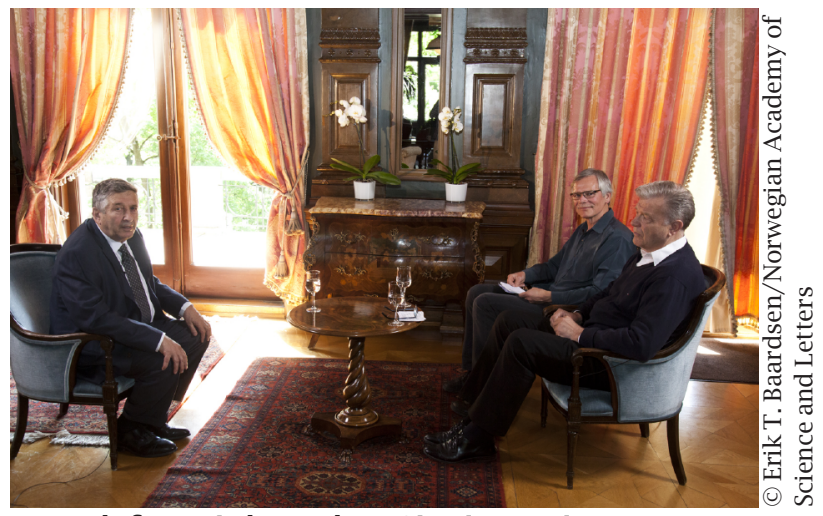

From left to right: Yakov Sinai, Martin Raussen, Christian Skau.

religious books and spend a lot of time doing this, which is conducive to learning. At that time, following the Jewish religion was strictly forbidden. People still did, however, but under very high pressure. If you do something under pressure, you work more. There is some kind of conservation law. This is my opinion of why Jews could succeed.

Raussen and Skau: You had to be much better in order to get the same opportunities?

Professor Sinai: I think it would be wrong to say that we had this feeling. We certainly tried to prepare for all exams and competitions. The result was not clear a priori, but there was always a hope that something could come out of it.

Raussen and Skau: Perhaps another reason is (especially under Stalin but also later) that a lot of very intelligent people were attracted to the natural sciences because there were fewer restrictions than in, say, history or political science....

Professor Sinai: That is certainly true. I can give you one example: at the time, Mech-Mat, the Faculty of Mechanics and Mathematics, had many graduate students that came from other countries. The rule was that they could only have advisors who were members of the Party. But there were students who wanted to work with Arnold, with me, or perhaps with some other people. The way out of this situation was the following: there were a number of people in the Party who became the students' official advisors, but the students actually worked with professors and mathematicians who were not members of the Party.

\section{East and West}

Raussen and Skau: You told us that you were not allowed to travel for many years, and this happened to a lot of Russian mathematicians at the time. Did these obstructions hamper or delay progress in science? Did it have the effect that Russian mathematics did not get recognition in the West that it deserved?

Professor Sinai: It is very difficult to answer your question, because you are asking what would have happened if something didn't happen. It is 
just impossible to say. It certainly caused harm, but it is not clear how big it was.

Raussen and Skau: Arnold was rather adamant about the lack of recognition. As a consequence of bad communication between East and West, results by Russian mathematicians during the isolation period were sometimes later rediscovered in the West. Therefore Russian mathematicians did not get the credit they deserved.

Professor Sinai: I have perhaps a special point of view concerning this. The question is whether some results can be stolen or not. My point of view, to which many people probably won't agree, is that if a result can be stolen, it is not a very good result.

Raussen and Skau: Tell us about the Landau Institute for Theoretical Physics in the Russian Academy of Sciences, your workplace for many years.

Professor Sinai: For many years the Landau Institute was the best institution in Russia. It was organized after Lev Landau's untimely death as a result of a car accident. Its director, I. M. Khalatnikov, had a remarkable talent to find gifted people all over Russia and to invite them to the institute. After several years the Landau Institute had a very strong group of physicists like Abrikosov, Gorkov, Dzyaloshinski, A. B. Migdal, Larkin, Zakharov, Polyakov, A. A. Migdal, and many others. The group of mathematical physicists was headed by S. P. Novikov and was much smaller.

It turned out that there was a big area of theoretical physics in which mathematicians and physicists could understand each other very well. They could even work on similar problems. Among these mathematicians I can name Novikov, Krichever, Khanin, Shabat, and Bogoyavlenskiy. Sometimes we invited physicists to explain to us their results in our seminars. The tradition of discussing problems of mutual interest still prevails.

Raussen and Skau: You moved in 1993 from the Landau Institute to Princeton University while still maintaining your position in Moscow. Why was it so attractive for you to go to the USA?

Professor Sinai: That is an easy question. First of all, I had many friends at Princeton. When we met we always had many points for discussion and common interests. Another reason was that many people had escaped from Russia so the situation there was no longer what it was before. In previous times everybody was in Moscow and Saint Petersburg, and you could call everyone to ask questions or to have discussions. Now that became impossible. The working conditions were better in the West and in particular at Princeton.

Raussen and Skau: You have now been in the USA for more than twenty years and you must know the American system almost as well as the Russian one. Could you tell us about how they compare from your perspective?

Professor Sinai: Concerning academic lives, it seems to me that they are more or less similar.
However, I must stress that I was never a member of any scientific committee at Mech-Mat at Moscow State University, and I was never invited to participate in any organizational meetings. Now I am chairman of the Scientific Council at the Institute of Transmission of Information.

\section{Teaching and Collaboration}

Raussen and Skau: You have been teaching courses and seminars for almost all your career. Do you have a particular technique or philosophy?

Professor Sinai: First of all, I like to teach undergraduate courses rather than graduate courses for the following reason: when you teach undergraduate courses you can easily see how your students become cleverer and more educated as they absorb new notions and connections and so on. When you teach graduate courses, the subject matter is usually a narrow piece of work, and students are mostly interested in some special issues that are needed for their theses. For me, that is less attractive.

My basic principle is as follows: if people do not understand my explanations, then this is my fault. I always ask students to ask questions as much as possible. Students who have asked me many questions during the lecture course have better chances for a good mark.

Raussen and Skau: You have an impressive list of students that have done well after graduation under your supervision. Grigoriy Margulis, just to mention one name, won the Fields Medal in 1978, and he will give one of the Abel lectures related to your work later this week....

Professor Sinai: I think the reason for this is not because of me but because of the types of problems we worked on. We did very interesting mathematics and formulated interesting problems that students were attracted to. This is my explanation. Many students preferred to work independently, and I was never against this.

Raussen and Skau: You are a very good example of the fact that mathematicians can flourish in late age as well. We came across a paper on number theory that you published this year together with two of your students. You have also published other papers related to number theory, so you must have kept an interest in that aspect of ergodic theory?

Professor Sinai: Yes, definitely. In the field we are working in there are many problems that are more natural for ergodic theory than for number theory. I don't want to be specific, but we had a paper that was more natural for an ergodic theorist than for a number theorist, so we could get the results more easily.

Raussen and Skau: Many joint papers appear on your list of publications. Apparently you like to have a lot of collaborators.

Professor Sinai: Well, I would say that they like it! And I'm not against it. It has never happened 
that I have asked someone to be my coauthor. I can only talk about some problems and explain why they are interesting.

But you are right, I have had many coauthors. I very much liked collaborating with Dong Li, who is now a professor at the University of British Columbia. When we work on the same problem we call each other many times a day. There are many others of my students with whom I liked to work. It's different to work with different people. Certainly I can work with Russian mathematicians as well as with mathematicians from other countries. Sometimes I like to work alone, but with age I need coauthors.

Raussen and Skau: You have only published one paper with Kolmogorov, but you have mentioned that you would have liked to publish more papers with him.

Professor Sinai: At a certain time, Kolmogorov decided that the Soviet Union did not have enough applied statistics. He worked on theoretical statistics and found many beautiful and deep results, but he was not satisfied with the fact that the theorems in applied statistics were not used for practical purposes. He found a problem related to the motion of the rotational axis of the earth that could be studied with the help of mathematical statistics. French observatories published data about the axis of rotation every two weeks, and Kolmogorov wanted to construct statistical criteria that could predict this motion. He wanted us to work on this problem and invited a very good geophysicist, Yevgeny Fyodorov, who was one of the main experts in this field. We were sitting there; Kolmogorov and Fyodorov were present. Kolmogorov said, "Look at these people; they prefer to write a paper for Doklady instead of doing something useful" (Doklady was the leading Russian journal). In our joint paper (by M. Arató, A. Kolmogorov, and me) written on this occasion, practically everything was done and written by Kolmogorov. Later, M. Arató wrote a big monograph on that subject.

In other cases, I often tried to explain my latest results to Kolmogorov. Sometimes his reaction was unexpected: "Why did you work on that problem? You are already a grown-up." But usually his reaction was very friendly. I regret very much that we never worked together; perhaps the reason is a difference in style.

Raussen and Skau: Wasn't it Kolmogorov who said that he spent a maximum of two weeks on a problem?

Professor Sinai: Kolmogorov used to stress that he did not have papers on which he worked for a long time. He mostly prepared his papers, including the proof and the text, in just two weeks, and this was a major difference in our approaches. Kolmogorov was a person with a strong temperament and he could not do anything slowly. I worked on some of my papers for years.

Raussen and Skau: He was a towering figure, not only in Russian mathematics but worldwide in the twentieth century.

Professor Sinai: Yes, definitely. Can I tell you one more story about him? It was when Kolmogorov was close to eighty. I asked him how it happened that he was a pure mathematician, even though he worked on concrete physical problems like turbulence. He answered that he was studying the results of concrete experiments. He had a lot of papers with results from experiments lying on the floor. He was studying them, and in this way he came up with his hypotheses on turbulence.

Raussen and Skau: So his intuition was motivated by physical considerations?

Professor Sinai: Yes. He subscribed to physical journals, and one could say he was into physics in a big way.

Raussen and Skau: Is that also true for you? Do you think mainly in terms of algebraic or analytic formulas? Or is it geometric intuition or even a mixture of all of that?

Professor Sinai: It depends on the problem. I can come to the conclusion that some problem must have a specific answer. I just told a journalist the story about a problem in which I knew there should be a definite answer. I worked on this problem for two years, and at the end of that time I discovered that the answer was one-half!

In general, I probably prefer to develop theories, sometimes to find the right concepts, rather than [to solve] specific problems.

Raussen and Skau: Have you had what we sometimes call a Poincaré moment, where all of sudden you see the proof?

Professor Sinai: Ideas often come unexpectedly, sometimes like revelations. But it happens only after a long period, maybe years, of difficult work. It did not happen while trying to find a taxi or something similar. It was very hard work for a long time, but then suddenly there was a moment where it became clear how the problem could be solved.

Raussen and Skau: If you yourself made a list of the results that you are most proud of, what would it look like?

Professor Sinai: I like all of them.

\section{Mountaineering}

Raussen and Skau: You mentioned Arnold, who died four years ago, an absolutely brilliant Russian mathematician. Arnold is, among many other things, known for his contributions to the so-called KAM theory. You both followed Kolmogorov's course and seminars in 1958. You told us that there was a close friendship already between your grandfathers. 


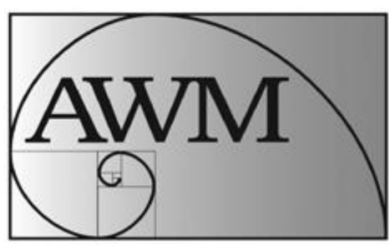

ASSOCIATION FOR WOMEN IN MATHEMATICS

\section{AWM Research Symposium 2015}

University of Maryland College Park April $11-12,2015$

PLENARY SPEAKERS:

Maria Chudnovsky, Columbia University

Ingrid Daubechies, Duke University

Jill Pipher, Brown University

Kathrin Wehrheim, UC Berkeley

SPECIAL SESSIONS on a wide-range of topics in pure and applied mathematics, statistics and mathematics education: Research from the "Cutting EDGE" ; Many facets of Probability ; Topics in Computational Topology and Geometry; Low-dimensional Topology; Number Theory, Mathematics at Government Labs and Centers; Graph Theory and Combinatorial Optimization; Symplectic Topology/Geometry; Harmonic Analysis, Signal Processing and Compressive Sensing; Algebraic Geometry; Mathematics Education; Statistics; PDEs in Continuum Mechanics

\section{POSTER SESSIONS, NETWORKING EVENT \& BANQUET}

For details on registration, housing, and the special sessions visit www.awm-math.org

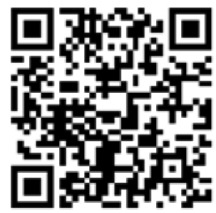

Sponsors and funders: University of Maryland, NSA and Microsoft Research. NSF support is expected but pending.
Both you and Arnold loved the outdoors and hiking. You once went to the Caucasus Mountains together, and you have to tell us the story about what happened when you stayed in the tents with the shepherds.

Professor Sinai: That is a very funny story. The weather was very bad; there was a lot of rain. We came to the shepherds' tent, and they let us in, and we could dry our clothes. We had lost our tent in the mountains, so we decided to go back to try to find it. We started to walk back, but these shepherds had some very big dogs-Caucasus dogs, a really big race. The shepherds weren't there any longer, and when the dogs found out that we were leaving, they surrounded us and started to bark ferociously. Arnold began to yell back with all the obscenities he knew, and the dogs did not touch him. But they attacked me. They didn't touch my skin, but they ripped my trousers apart. Finally, the shepherds came back and we were saved.

Raussen and Skau: We would like to ask one final question that has nothing to do with mathematics. You have certainly focused on mathematics during your life, but surely you have developed other interests also?

Professor Sinai: I was interested, especially in former years, in many different sports. I was a volleyball player and I liked to ski, both downhill and cross-country. I also liked mountaineering, but I cannot say I was a professional. I climbed often with a close friend of mine, Zakharov, who worked on integrable systems. We were climbing in the mountains together, and once we were on a very difficult 300-meter-long slope, which took us four hours to get down from! We had to use ropes and all sorts of gear. Nowadays, my possibilities are more limited.

Raussen and Skau: Thank you very much for this most interesting conversation. We would like to thank you on behalf of the Norwegian, the Danish, and the European Mathematical Societies.

Professor Sinai: Thank you very much. 